\title{
Analysis of maximum joint moment during infant lifting-up motion
}

\author{
Ji-Won Kim ${ }^{\mathrm{a}, \mathrm{b}}$, Gwang-Moon Eom ${ }^{\mathrm{a}, \mathrm{b}, 1}$ and Yu-Ri Kwon ${ }^{\mathrm{a}, \mathrm{b}, 1, *}$ \\ ${ }^{\mathrm{a}}$ BK21 Plus Research Institute of Biomedical Engineering, Konkuk University, Chungju, Korea \\ ${ }^{\mathrm{b}}$ Department of Biomedical Engineering, Konkuk University, Chungju, Korea
}

\begin{abstract}
.
BACKGROUND: Infant care activities can induce musculoskeletal disease. However, little is known about the biomechanical joint load during lifting-up of an infant.

OBJECTIVE: The aim of this study was to investigate normalized maximum moment during lifting-up of infant dummies weighing $4.6 \mathrm{~kg}, 7.6 \mathrm{~kg}$, and $9.8 \mathrm{~kg}$.

METHODS: Six healthy young subjects participated in our study. All subjects performed lifting-up activities of dummies to shoulder height with their feet apart and natural postures in their comfortable speed. Three-dimensional reflective marker trajectories and ground reaction forces were used as input to calculate joint moments using a full body musculoskeletal model. Joint moments were normalized by each subject's body mass. Friedman's test was performed to compare mean differences of normalized joint moments for lifting up three dummy weights.

RESULTS: Lumbar joint had the greatest normalized joint moment. Lumbar and hip extension moments were significantly increased with dummy weight $(P<0.05)$. In contrast, knee extension and ankle plantarflexion moment were not significantly affected by dummy weight $(P>0.05)$.

CONCLUSIONS: These results indicate that the lumbar joint plays the most important role in infant lifting-up motion and that the load of lumbar and hip joint should be reduced when lifting a heavier infant. These results could contribute to the development of an effective lifting strategy and an assisting device for lifting an infant.
\end{abstract}

Keywords: Joint moment, back pain, inverse dynamic simulation, infant care, lifting-up motion

\section{Introduction}

Infant care activities, i.e., lifting-up, holding, and carrying infant, are physical tasks that can pose challenges to parents. They commonly induce mechanical load on the musculoskeletal system due to repetitive strain and inappropriate postures [1,2]. Specifically, people who care infant are required to regularly lift up and carry infants. They spend a lot of activities such as squatting, kneeling, and sitting on a floor or low couch with mechanical load for a long period of time [3]. They might use a poor infant lifting technique throughout the day. For example, a stooped position with straight knees and a bent back for lifting an infant from the floor can induce strain of the soft non-muscular tissue of the back that supports the infant [4]. Furthermore, a correct lifting movement of an infant is difficult because the infant is possibly in constant and unpredictable motion during the lift [5]. This also results in lifting an infant

\footnotetext{
${ }^{1}$ These authors contributed equally to this work as co-corresponding authors.

${ }^{*}$ Corresponding author: Yu-Ri Kwon, Department of Biomedical Engineering, Konkuk University, 268 Chungwon-daero Chungju-si Chungcheongbuk-do 27478 Korea. Tel.: + 8243840 4953; Fax: +82 43851 0620; E-mail: $07167 @$ daum.net.
} 
Table 1

\begin{tabular}{lrc}
\multicolumn{3}{c}{ Demographic characteristics of subjects } \\
\hline Variables & Mean & SD \\
\hline Age (years) & 24.8 & 0.5 \\
Height $(\mathrm{cm})$ & 171.8 & 2.6 \\
Weight $(\mathrm{kg})$ & 72.6 & 6.5
\end{tabular}

in awkward positions that are biomechanically stressful $[5,6]$. Thus, infant care activities can result in musculoskeletal diseases. For example, it has been reported that $25 \%$ of women after childbirth suffer from a low back pain and a pelvic girdle pain [7]. Especially, abnormal postures and positions during long infant care periods have been widely identified as contributing factors to lower back pain [8]. Also, 49\% of back injuries have been reported to be associated with lifting an infant [6]. In addition, many research studies have demonstrated that carrying a load such as an infant in the front with arms can cause lumbar lordosis $[9,10]$, which can lead to lower back pain [11]. Furthermore, it has been reported that infant carrying is associated with increased trunk muscle activity $[10,12,13]$ that can induce reduced muscular endurance [14].

It is important to understand biomechanical implications of infant care movements for suggesting an effective infant care strategy. Therefore, some studies have investigated quantitative biomechanical strategy during infant care activities such as carrying in-arms or sling-based infant carrying. Schmid et al. have investigated effects of different sling-based infant carrying methods using motion capture camera and electromyography [15]. They suggested that carrying an infant alternating on preferred and non-preferred sides using a sling could help prevent musculoskeletal diseases resulting from paraspinal muscle hyperactivation and excessive lumbar hyperextension. Williams et al. have investigated lower extremity joint moments during carrying an infant using a baby carrier [16]. They demonstrated that a baby carrier could decrease the loading knee abduction moment by $8.7 \%$ and the loading knee extension moment by $16.7 \%$ compared to an in-arm carriage.

However, previous studies have only focused on biomechanical analysis about infant carrying. Studies investigating biomechanical kinetics of human joints during lifting-up an infant have not been reported yet. In addition, increased weight of an infant might affect biomechanical joint load. Therefore, the aim of this study was to perform kinetic analysis during lifting-up of infant dummies with various weights.

\section{Method}

\subsection{Subjects}

Six healthy young men were recruited from Konkuk University for this study (Table 1). Subjects with any diseases (i.e., lower limb injury, musculoskeletal disease, and neurological disorders) that might affect their activities of lifting load were excluded. Before participation, the aim of the study was explained to all subjects. This study was approved by the ethics committee of Konkuk University. Written informed consent was obtained from each subject prior to main measurements.

\subsection{Experiments and analysis}

Three-dimensional motion analysis data were acquired using an Eagle six-camera system (Motion Analysis Corp., Santa Rosa, CA, USA) for assessment of movement patterns during lifting an infant dummy. Thirty-three retroreflective markers were attached onto anatomic landmarks of each subject 

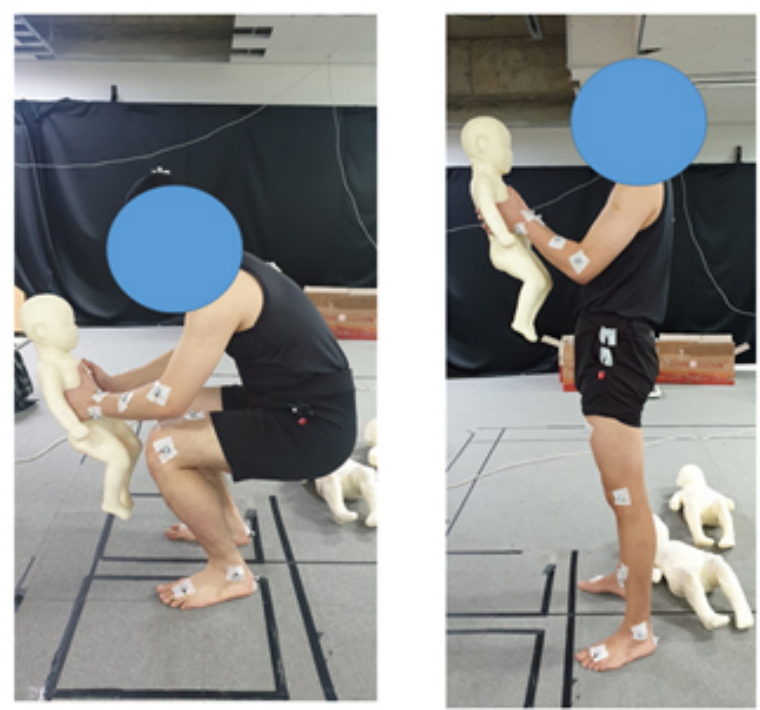

Fig. 1. Placement of reflective markers on anatomic landmarks for motion capture during lifting up an infant dummy.

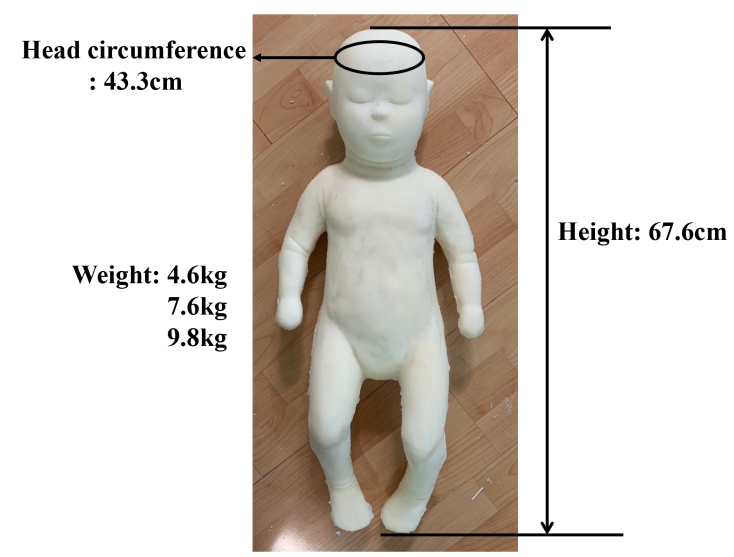

Fig. 2. The specification of the developed infant dummy.

according to the Helen Hayes marker set as shown in Fig. 1. Marker data were collected at $120-\mathrm{Hz}$ sampling rate with an EvaRT software (Motion Analysis Corp., Santa Rosa, CA, USA). Two force plates (OR-7-2000, $464 \mathrm{~mm} \times 508 \mathrm{~mm} \times 84 \mathrm{~mm}$; Advanced Mechanical Technology, Watertown, NY, USA) were synchronized with the camera system to acquire ground reaction force (GRF) and center of pressure (COP). Both GRF and COP data were sampled at a sampling rate of $1200-\mathrm{Hz}$ with a data acquisition board.

As shown in Fig. 2, infant dummies weighing $4.6 \mathrm{~kg}, 7.6 \mathrm{~kg}$, and $9.8 \mathrm{~kg}$ were used instead of real infants. These weights $(4.6 \mathrm{~kg}, 7.6 \mathrm{~kg}$, and $9.8 \mathrm{~kg}$ ) of infant dummies used in this study were selected as average weights of 1-month, 6-month, and 12-month infants in Korea, respectively [18]. Previous studies have also used infant dummies for biomechanical analysis during infant care $[15,16]$. Furthermore, it has been reported that carrying an infant is not significantly different from carrying an infant dummy during walking [9]. 


\section{Dummy position 1}

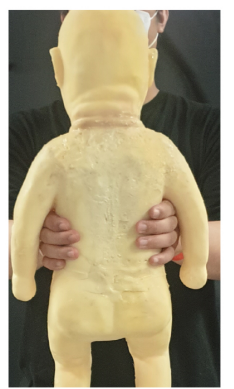

Dummy position 2 (lying dymmy)

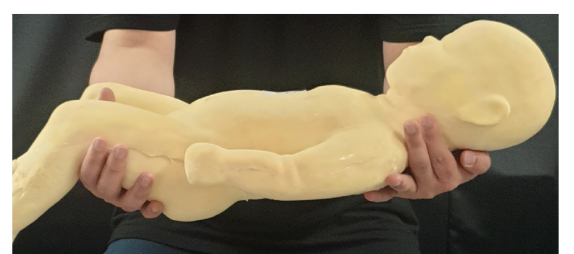

Fig. 3. Definitions of dummy positions (right: lifting a lying dummy; left: lifting armpit of the dummy).
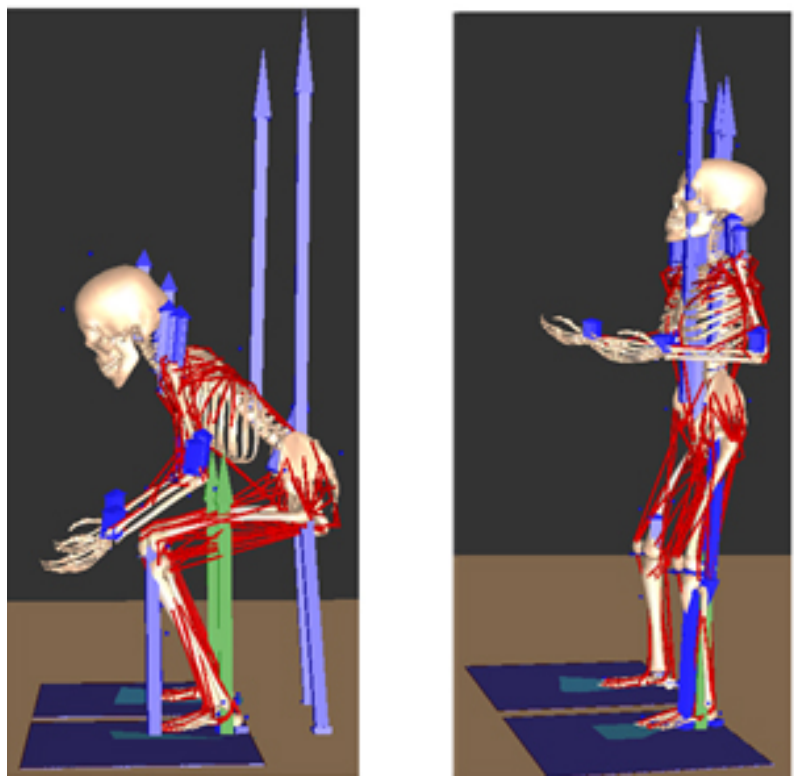

Fig. 4. Inverse dynamic simulation for calculating joint moments on lower and upper extremities during a lifting-up motion.

All subjects were asked to lift infant dummies using two different lifting techniques (dummy positions 1 and 2). As shown in Fig. 3, dummy positions 1 and 2 were defined as lifting armpit of dummy and lifting a lying dummy, respectively. Although 1-month dummy in dummy position 1 is unrealistic in infant care motions because a baby cannot keep his or her head up, we included this dummy corresponding to the weight of a one-month-old baby in order to investigate joint moments according to increasing dummy weight. All subjects were instructed to lift up each dummy to shoulder height with their feet apart and natural postures in their comfortable speed.

Joint moments on shoulder, lumbar, hip, knee, and ankle were calculated using an inverse dynamics simulation method. Briefly, a software for interactive musculoskeletal modeling (SIMM) (MuscouloGraphics Inc., Motion Analysis Corp., Santa Rosa, CA, USA) was used to compute joint moments via an inverse dynamic simulation based on full body model kinematics and ground reaction forces as shown in Fig. 4. Maximum joint moments were normalized by each subject's body mass. Joint moments are known to be influenced by weight. Common approaches for normalization of joint moments include dividing the joint moment by body mass [19]. Non-parametric Friedman's test was performed to determine main 

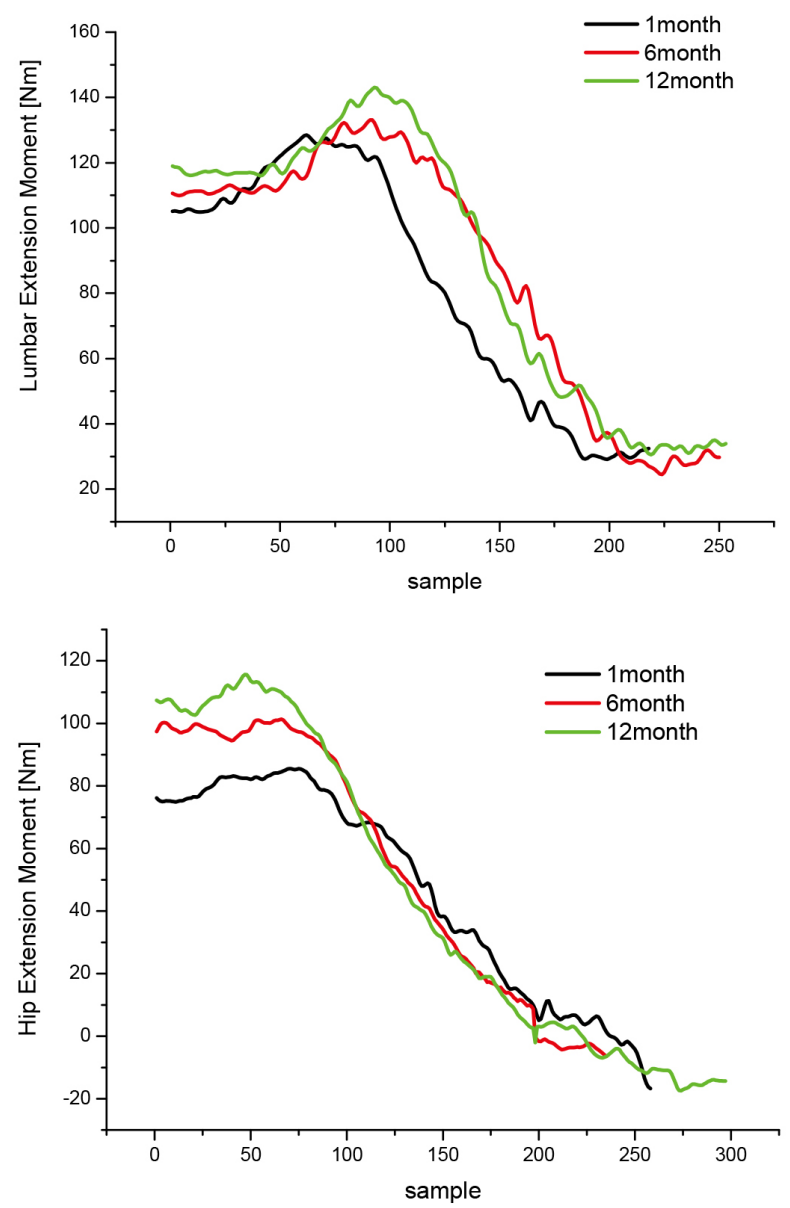

Fig. 5. Representative joint moment trajectories according to dummy weight.

effects of dummy weight on normalized maximum moment in each joint. When a significant main effect was found, Wilcoxon signed-rank test was used in order to compare maximal joint moment of each dummy weight. Statistical analysis was performed using SPSS ver. 16 for Windows (SPSS Inc., Chicago, IL, USA). The significant level was defined at $p<0.05$.

\section{Results}

Figure 5 shows trajectories of representative lumbar and hip joint moments according to dummy weight. The maximum extension moment at both lumbar and hip joints increased with increasing dummy weight.

Table 2 presents results of Friedman's test comparing normalized joint moments with increased infant dummy weight. Lumbar joint had the greatest normalized joint moment in most dummy types and both lifting techniques (dummy positions 1 and 2). In both dummy positions 1 and 2, main effects of dummy type (weight) were significant in shoulder, lumbar joints, and hip joints $(P<0.01)$. In contrast, there were no significant main effects in knee or ankle joints $(P>0.05)$.

Figures 6 and 7 show post hoc results of normalized maximum joint moments according to dummy types in each joint. Shoulder, lumbar, and hip extension moments were increased with increasing dummy 
Table 2

Normalized maximum joint moment according to increased weight in each joint

\begin{tabular}{|c|c|c|c|c|c|c|c|c|c|}
\hline \multirow{2}{*}{\multicolumn{2}{|c|}{ Variables }} & \multicolumn{4}{|c|}{ Dummy position 1} & \multicolumn{4}{|c|}{ Dummy position 2} \\
\hline & & \multicolumn{3}{|c|}{ Dummy type (weight) } & \multirow{3}{*}{$\begin{array}{l}\text { Signifi- } \\
\text { cance }\end{array}$} & \multicolumn{3}{|c|}{ Dummy type (weight) } & \multirow{3}{*}{$\begin{array}{c}\text { Signifi- } \\
\text { cance }\end{array}$} \\
\hline & & 1-month & 6-month & 12-month & & 1-month & 6-month & 12-month & \\
\hline & & Mean (SD) & Mean (SD) & Mean (SD) & & Mean (SD) & Mean (SD) & Mean (SD) & \\
\hline Norn & Shor & $0.2(0.10)$ & $0.28(0.14)$ & $0.34(0.21)$ & 0.002 & $0.21(0.08)$ & 0.29 & & 0.002 \\
\hline Maximum & Lumbar & $1.66(0.14)$ & $1.84(0.13)$ & 2.02 & 0.0 & $1.63(0.13)$ & 1.79 & 1.9 & 0.002 \\
\hline Joint & Hip & $1.41(0.14)$ & $1.74(0.35)$ & $2.02(0.30)$ & 0.002 & $1.33(0.17)$ & $1.52(0.28)$ & $1.84(0.30)$ & 0.002 \\
\hline moment & Knee & $1.23(0.51)$ & $1.45(0.48)$ & $1.31(0.41)$ & 0.115 & $1.57(0.26)$ & $1.59(0.39)$ & $1.67(0.45)$ & 0.513 \\
\hline$(\mathrm{Nm} / \mathrm{kg})$ & Ankle & $0.61(0.23)$ & $0.73(0.37)$ & $0.85(0.42)$ & 0.513 & $0.62(0.31)$ & $0.63(0.31)$ & $0.95(0.45)$ & 0.115 \\
\hline
\end{tabular}
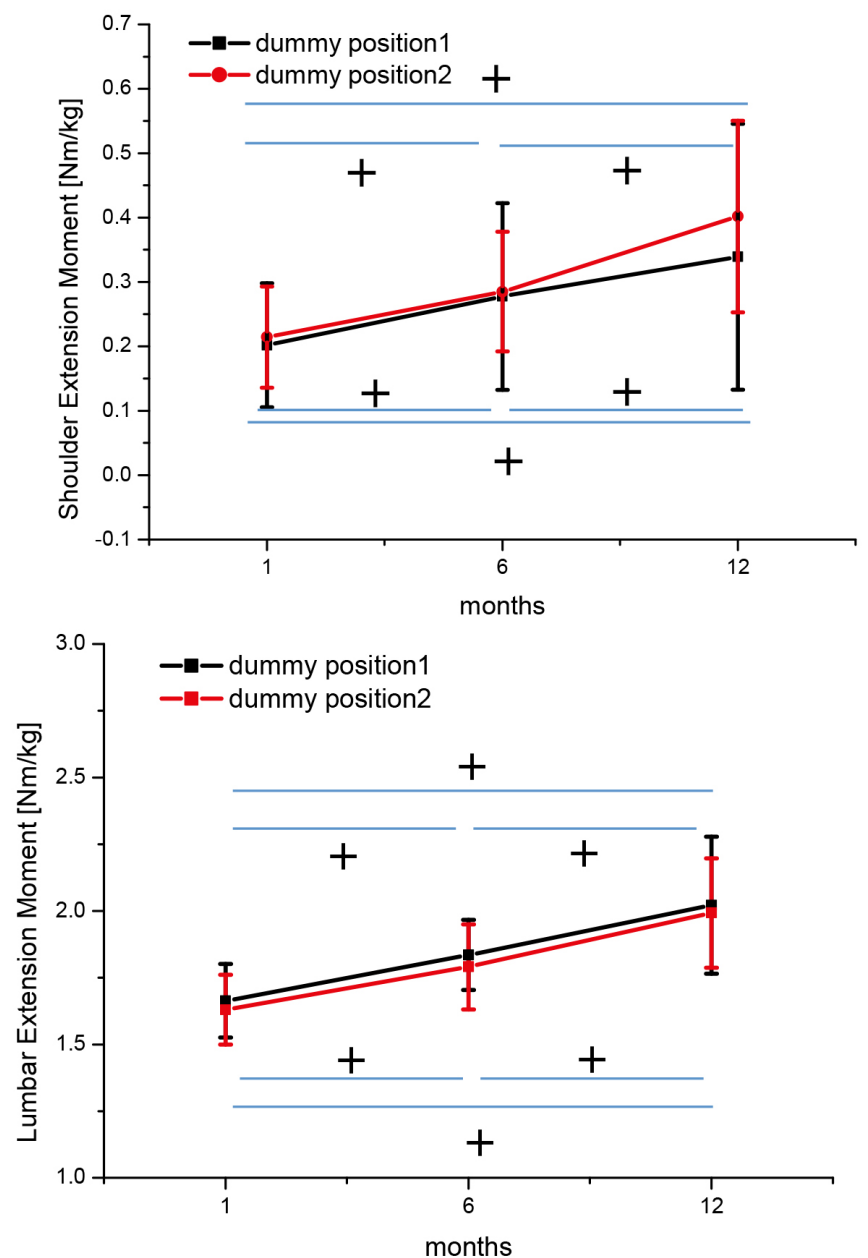

Fig. 6. Post hoc test results of normalized maximum joint moment during the lifting-up motion in shoulder and lumbar joints (+: $P<0.05)$.

weight in both dummy positions 1 and 2 . Specifically, there was a significant weight effect, with motion for lifting-up 12-month infant dummy having a significantly greater moment than the motion for lifting-up 1 -month or 6-month infant dummy $(P<0.05)$. Similarly, maximum moments for lifting-up 6-month 

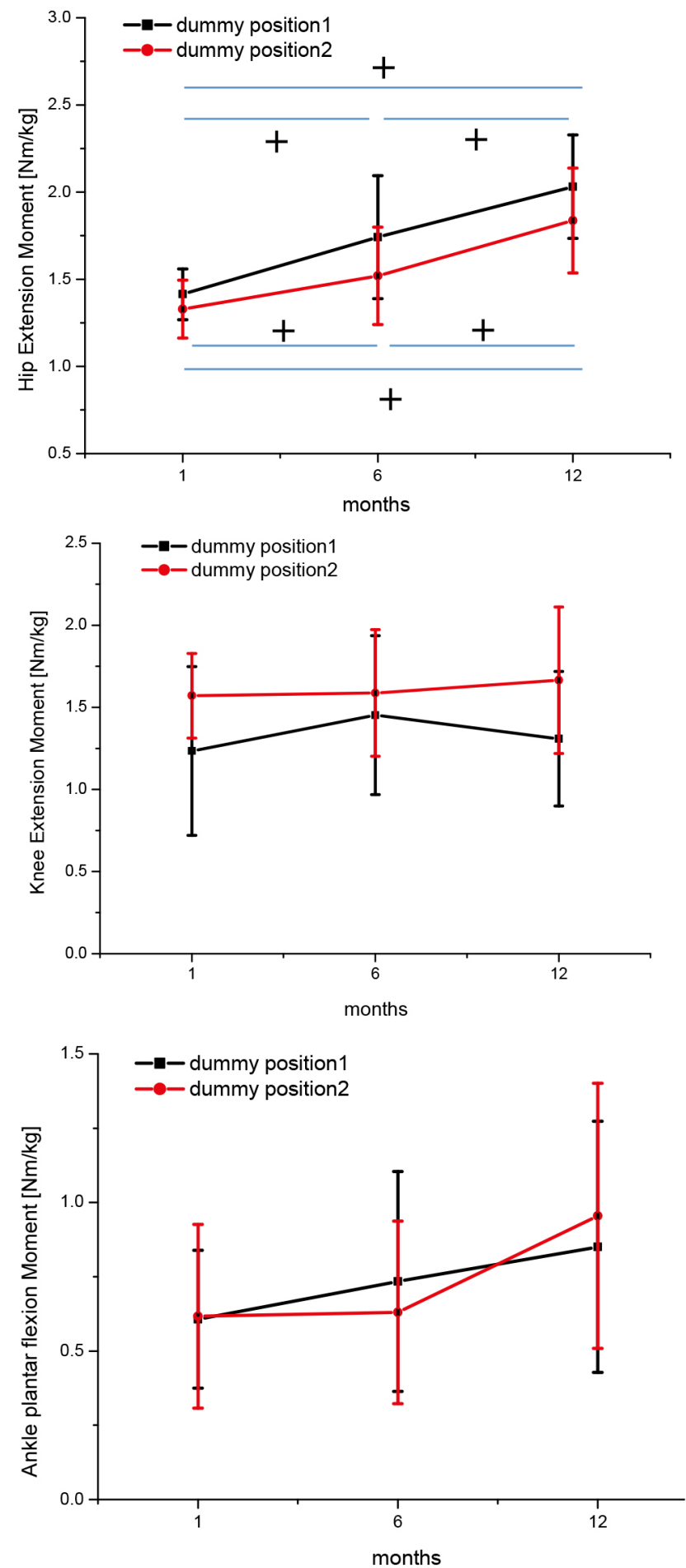

Fig. 7. Post hoc test results of normalized joint moment during the lifting-up motion in lower extremities $(+: P<0.05)$. 
infant dummy were significantly greater than those for lifting-up 1-month infant dummy $(P<0.05)$. In contrast, knee extension moment and ankle plantarflexion moment did not change significantly with increasing dummy weight.

\section{Discussion}

Little is known about the kinetic characteristics during lifting-up of an infant. This study investigated normalized maximum joint moments during lifting-up of infant dummies with various weights. The main findings of this study were as follows. First, lumbar joint generated the greatest moment during lifting-up of an infant dummy. Second, normalized maximum joint moment in lumbar and hip extension motions significantly increased with increasing dummy weight, whereas ankle and knee joints were not significantly affected by infant dummy weight. Third, shoulder extension moment increased with increasing dummy weight only in dummy position 2 .

In this study, lumbar extension moment tended to be greater than other joint moments (Table 2). This tendency was found for all dummy weights and both dummy positions. This indicates that the lumbar extension moment play the most important role in a lifting-up motion. This result might be associated with the occurrence of low back pain and back injuries during infant care activities $[6,8]$. Our results are in agreement with previous similar studies showing that the greatest lumbar joint moment is generated in all load types during both stoop and squat lifting motions [17].

Increase of normalized maximum moment by dummy weight was prominent in lumbar and hip joints (Figs 6 and 7). Normalized maximum moment was significantly increased with increasing infant dummy weight (in the order of 1-month, 6-month, and 12-month infant dummies, $P<0.05$ ). This indicates that the heavier an infant, the greater the load on the lumbar and hip joints, which may result in occurrence of low back pain and/or pelvic girdle pain. In fact, it has been reported that $25 \%$ of women after childbirth suffer from a low back pain or pelvic girdle pain [7]. Shoulder extension moment was also significantly increased with increasing infant dummy weight in both dummy positions 1 and 2 . However, the shoulder joint moment was much smaller than lumbar and hip joint moments.

On the other hand, ankle and knee joint moment showed no significant changes with increasing infant dummy weight (Table 1). This means that ankle and knee joints had similar normalized maximum joint moment regardless of dummy weight. The reason for significant main effects on lumbar and hip joints might be because the center of mass (COM) of the body moved to the anterior with an increase of dummy weight. That is, greater moment might have been generated at lumbar and hip joints to overcome body COM moving to the anterior with a heavier infant dummy. In contrast, ankle and knee joints have less effects on the change of COM. Our results suggest that an assisting aid or device than can reduce increased load on lumbar and hip joints should be developed in order to prevent low back pain and pelvis girdle pain.

The normalized maximum moment between dummy positions 1 and 2 was prominent in hip and knee joints. Specifically, hip joint moment during lifting for dummy position 1 was greater than that during lifting for dummy position 2 . This result is attributed to the difference of maximum hip flexion angle due to different dummy positions during lifting-up of a dummy. Actually, the maximum hip flexion angle for dummy position 1 was significantly greater than that for dummy position $2(P<0.05)$, which was not shown in the result. That is, when lifting a dummy, a larger hip joint angle can induce the body's COM to move more backward. As a result, the hip joint moment might be greater to overcome the increased force exerted on the hip joint by the moved COM. In contrast, knee joint moment during lifting for dummy position 2 was greater than that during lifting for dummy position 1 . This might also be associated 
with COM moving backward. COM moving backward could induce smaller knee extension moment because force exerted on the knee joint was decreased by the moved COM. The normalized maximum moment for dummy positions 1 and 2 was similar between lumbar and shoulder joints because kinematic characteristics of upper extremities were not significantly different between the two dummy positions.

Many studies have reported that infant care activities can induce mechanical load on musculoskeletal systems. Mechanical loading has been identified as an important risk factor of musculoskeletal diseases such as low back pain [1,2]. Although biomechanical analysis during an infant carrying motion has been performed, some studies have focused on lower extremities [15] with only a single dummy weight $[15,16]$. Furthermore, there has been no study on joint loading during lifting motion of an infant. This study demonstrated that lumbar extension load had the greatest influence on lifting-up of an infant. Moreover, lumbar and hip extension loads were more prominently influenced by heavier infants such as a 12-monthold infant. Therefore, further studies are needed to develop an effective lifting strategy so that load on lumbar and hip joints could be reduced.

This study has some limitations. Limitations and future directions of this study are shown as follows: (1) This study focused on only two dummy-holding positions. In real life, holding positions for a baby are various during baby-care activities. For example, parents can hold an infant in their chest with both arms. Also, they can sustain uncomfortable postures for baby feeding. Therefore, more infant-holding positions should be used for more realistic kinetic analysis in infant care activities. (2) When lifting an infant after bathing, an infant is usually lifted far away from parent's body. The distance between an infant and the parent's body during lifting an infant can affect joint moments. For example, if parents lift an infant far away from their body, a greater lumbar joint moment might be produced. Therefore, the joint moment depending on the distance from an infant should be analyzed in a further study. (3) In this study, a small number of subjects participated. A larger population of participants should be included considering gender and age in a further study.

\section{Conclusions}

Normalized maximum joint moments at the shoulder, lumbar, ankle, knee and hip joints during liftingup of infant dummies with various weights were investigated. The lumbar extension moment was found to be the greatest during lifting-up of an infant. Older (heavier) infant had a greater influence on lumbar and hip joints. These findings provide new insight into infant care motion. These results could contribute to the prevention of musculoskeletal diseases due to infant care activities by developing an effective lifting strategy and an assisting device to reduce loads on lumbar and hip joints.

\section{Acknowledgments}

This work was supported by the National Research Foundation of Korea (NRF) grant funded by the Korea government (MSIT) (No. 2019R1C1C1008209).

\section{Conflict of interest}

None to report. 


\section{References}

[1] Sanders MJ, Morse T. The ergonomics of caring for children: An exploratory study. American Journal of Occupational Therapy. 2005 May-Jun; 59(3): 285-95.

[2] Borg-Stein J, Dugan SA. Musculoskeletal disorders of pregnancy, delivery and postpartum. Phys Med Rehabil Clin N Am. 2007 Aug; 18(3): 459-76, ix.

[3] Calabro KS, Bright KA, Cole FL, Mackey T, Lindenberg J, Grimm A. Child care work. Organizational culture and health and safety. AAOHN J. 2000 Oct; 48(10): 480-6.

[4] Griffin SD, Price VJ. Living with lifting: mothers perceptions of lifting and back strain in childcare. Occupational Therapy International. 2000; $7:$ 1-20.

[5] Owen BD. Intervention for musculoskeletal disorders among child-care workers. Pediatrics. 1994 Dec; 94(6 Pt 2): 1077-9.

[6] Brown MZ, Gerberich SG. Disabling injuries to childcare workers in Minnesota, 1985 to 1990. An analysis of potential risk factors. J Occup Med. 1993 Dec; 35(12): 1236-43.

[7] Wu WH, Meijer OG, Uegaki K, Mens JM, van Dieen JH, Wuisman PI, et al. Pregnancy-related pelvic girdle pain (PPP), I: Terminology, clinical presentation, and prevalence. Eur Spine J. 2004 Nov; 13(7): 575-89.

[8] Phillips JA, Forrester B, Brown KC. Low back pain: prevention and management. AAOHN J. 1996 Jan; 44(1): 40-51; quiz 2-3.

[9] Junqueira LD, Amaral LQ, Iutaka AS, Duarte M. Effects of transporting an infant on the posture of women during walking and standing still. Gait Posture. 2015 Mar; 41(3): 841-6.

[10] Anderson AM, Meador KA, McClure LR, Makrozahopoulos D, Brooks DJ, Mirka GA. A biomechanical analysis of anterior load carriage. Ergonomics. 2007 Dec; 50(12): 2104-17.

[11] Masharawi Y, Dar G, Peleg S, Steinberg N, Medlej B, May H, et al. A morphological adaptation of the thoracic and lumbar vertebrae to lumbar hyperlordosis in young and adult females. Eur Spine J. 2010 May; 19(5): 768-73.

[12] Motmans RR, Tomlow S, Vissers D. Trunk muscle activity in different modes of carrying schoolbags. Ergonomics. 2006 Feb 10; 49(2): 127-38.

[13] Svenningsen FP, Kaalund E, Christensen TA, Helsinghoff PH, Gregersen NY, Kersting UG, et al. Influence of anterior load carriage on lumbar muscle activation while walking in stable and unstable shoes. Hum Mov Sci. 2017 Dec; 56(Pt B): 20-8.

[14] Gutke A, Ostgaard HC, Oberg B. Association between muscle function and low back pain in relation to pregnancy. J Rehabil Med. 2008 Apr; 40(4): 304-11.

[15] Schmid S, Stauffer M, Jager J, List R, Lorenzetti S. Sling-based infant carrying affects lumbar and thoracic spine neuromechanics during standing and walking. Gait Posture. 2019 Jan; 67: 172-80.

[16] Williams L, Standifird T, Madsen M. Effects of infant transportation on lower extremity joint moments: Baby carrier versus carrying in-arms. Gait Posture. 2019 May; 70: 168-74.

[17] Have AV, Rossom SV, Jonkers I. Squat Lifting Imposes Higher Peak Joint and Muscle Loading Compared to Stoop Lifting. Appl Sci. 2019; 9(18): 3794.

[18] Korean National Growth Charts for children and adolescents, Korea Centers for Disease Control and Prevention, 2017.

[19] Winter DA. The biomechanics and motor control of human gait: normal, elderly, and pathological: Waterloo Press, Waterloo, 2001 